\title{
Influence of mass diffusion in sea ice dynamical models
}

Article

Accepted Version

Wilchinsky, A. V. and Feltham, D. L. (2004) Influence of mass diffusion in sea ice dynamical models. Journal of Physical Oceanography, 34 (6). pp. 1468-1475. ISSN 0022-3670 doi: https://doi.org/10.1175/15200485(2004)034<1468:IOMDIS>2.0.CO;2 Available at https://centaur.reading.ac.uk/35293/

It is advisable to refer to the publisher's version if you intend to cite from the work. See Guidance on citing.

Published version at: http://dx.doi.org/10.1175/1520-0485(2004)034<1468:IOMDIS>2.0.CO;2

To link to this article DOI: http://dx.doi.org/10.1175/1520-

0485(2004)034<1468:IOMDIS>2.0.CO;2

Publisher: American Meteorological Society

All outputs in CentAUR are protected by Intellectual Property Rights law, including copyright law. Copyright and IPR is retained by the creators or other copyright holders. Terms and conditions for use of this material are defined in the End User Agreement.

www.reading.ac.uk/centaur 
Central Archive at the University of Reading

Reading's research outputs online 


\title{
Influence of Mass Diffusion in Sea Ice Dynamical Models
}

\author{
AleXANDer V. WilchinsKY* AND Daniel L. Feltham \\ Centre for Polar Observation and Modelling, Department of Space and Climate Physics, University College London, \\ London, United Kingdom
}

9 July 2003 and 1 December 2003

\begin{abstract}
The mixing of floes of different thickness caused by repeated deformation of the ice cover is modeled as diffusion, and the mass balance equation for sea ice accounting for mass diffusion is developed. The effect of deformational diffusion on the ice thickness balance is shown to reach $1 \%$ of the divergence effect, which describes ridging and lead formation. This means that with the same accuracy the mass balance equation can be written in terms of mean velocity rather than mean mass-weighted velocity, which one should correctly use for a multicomponent fluid such as sea ice with components identified by floe thickness. Mixing (diffusion) of sea ice also occurs because of turbulent variations in wind and ocean drags that are unresolved in models. Estimates of the importance of turbulent mass diffusion on the dynamic redistribution of ice thickness are determined using empirical data for the turbulent diffusivity. For long-time-scale prediction ( $\gg 5$ days), where unresolved atmospheric motion may have a length scale on the order of the Arctic basin and the time scale is larger than the synoptic time scale of atmospheric events, turbulent mass diffusion can exceed $10 \%$ of the divergence effect. However, for short-time-scale prediction, for example, 5 days, the unresolved scales are on the order of $100 \mathrm{~km}$, and turbulent diffusion is about $0.1 \%$ of the divergence effect. Because inertial effects are small in the dynamics of the sea ice pack, diffusive momentum transfer can be disregarded.
\end{abstract}

\section{Introduction}

The sea ice cover of the earth's polar oceans is a mosaic of floes (typical horizontal floe length scale is $0.1-10 \mathrm{~km}$ and typical depth is $1-10 \mathrm{~m})$. These floes are separated by approximately rectilinear regions of ocean or thin ice (leads) with widths typically smaller than the local floe span and lengths spanning many floes. To date, almost all models of sea ice dynamics have been developed for length scales typically 100-1000 times the dimensions of a single floe, and the sea ice pack is modeled as a continuum. These continuum models, for example, Coon et al. (1974), Hibler (1979), Tremblay and Mysak (1997), and many others, consist of equations describing local conservation of mass and momentum in continuous form. Because most of the sea ice pack has a high ice concentration (defined as the fractional area covered in sea ice in a given region),

\footnotetext{
* On a leave from Institute of Mathematics and Mechanics, Kazan State University, Kazan, Republic of Tatarstan, Russia.
}

Corresponding author address: A. Wilchinsky, CPOM, Department of Space and Climate Physics, UCL, Gower Street, London WC1E 6BT, United Kingdom.

E-mail: aw@cpom.ucl.ac.uk which typically ranges between $75 \%$ and over $95 \%$ in the central Arctic, the motion of assemblies of floes resembles that of granular materials. This is because significant momentum transfer primarily occurs at floefloe contacts rather than in the ocean between the floes (see, e.g., Tremblay and Mysak 1997). Floe-floe interaction is dominated by friction at the floes edges and irreversible failure (flow) due to sliding and rafting/ ridging.

Consider the following experiment: One-half of a rectangular region filled with white checkers, and the other with black checkers, is subjected to a slow deformation that finally leads to mixing of the checkers (see Fig. 1). The mixing of the checkers (floes) occurs because deformation causes them to move past each other in the direction determined by the angle between the checker centers and the strain direction, which practically is a random value and therefore can be described using a diffusion equation. We refer to this mixing as deformational diffusion. Diffusion in real sea ice is demonstrated, for example, by the fact that the remains of the American Ice Station Charley were found in 1966 north of Wrangel Island dispersed $50 \mathrm{~km}$ from each other (Litinsky 1968).

Turbulent eddies in the atmosphere and ocean exert tractions on the ice cover that cause sea ice to mix, or 

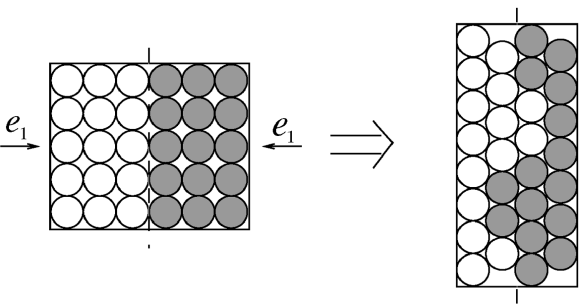

FIG. 1. An experiment with checkers showing diffusion during deformation. The checkers are confined by a rectangular frame that preserves the rectangular form of the region under deformation (pure shear). After the $50 \%$ deformation, the checkers are exchanged across the (dashed) areal line.

diffuse (Gudkovich 1979). Since not all scales of atmospheric and oceanic turbulence are resolved in numerical simulations of sea ice using coupled atmosphere-ice-ocean models (Holland 2001), turbulent diffusion should be assessed and, if significant, parameterized. Evidently, unresolved scales depend on the process under consideration: If the day-to-day dynamics of sea ice is modeled using real atmospheric circulation data, then the unresolved scales are determined by the discretization length and time step, and the primitive variables (ice thickness, concentration, and velocity) can be considered as locally averaged. If long-time-scale processes are considered, atmospheric instability and unpredicted evolution of boundary conditions results in large-scale turbulent eddies that cannot be explicitly taken into account and should be modeled as large-scale turbulent diffusion. In this case the primitive variables can be understood as Reynolds-averaged fields.

The effect of sea ice diffusion on the trajectory of a floe was studied by Colony and Thorndike (1985) who assumed an isotropic Gaussian relative displacement of the floe with regard to its mean displacement and found the mean age of the ice in the Arctic basin to be up to 7 years. Later, Thorndike (1986) studied separation between two floes due to diffusion in sea ice, which can be used to estimate the effect of the dispersion of pollutants. He demonstrated the deformational diffusion effect by constructing a simple model describing a sequence of random cracks splitting a region of sea ice into two parts moving past each other, which finally leads to significant mixing of the whole region under consideration. In order to estimate the importance of the process, analytical formulas for the relative displacement in isotropic turbulent diffusion and buoy motion data were used. This showed that, although on a small time scale the effect is usually negligible, on the time scale of a decade, considered as the time scale of a floe life, the process can lead to a significant separation between two initially close floes.

Although on large time scales the effect of sea ice diffusion on floe separation was shown by Thorndike (1986) to be important, its effect on the transfer of mass and momentum has not yet been estimated. Moreover, while the variance of relative displacement found from observations differs from their analytical counterpart found by considering homogeneous isotropic turbulence, it has not been shown if deformational diffusion contributes to this difference. If there is a gradient of the sea ice thickness, then both types of diffusion, deformational and turbulent, cause exchange of floes of different thicknesses that leads to a diffusive mass and thickness flux through a surface with zero mean velocity across it. In particular, if the black checkers in the above experiment had larger thickness than the white checkers, then there would be a diffusional mass transfer through the central line initially separating the different checkers from each other.

The balance equations for sea ice have been extensively studied (e.g., Gray and Morland 1994). However, the possible importance of deformational floe diffusion on the mass and momentum balance has not yet been treated on a continuum level. While turbulent diffusion of ice thickness was recently considered by Holland (2001) (he used a cavitating fluid sea ice rheology; see Flato and Hibler 1992), its importance was not explicitly treated and values of diffusivity were not calculated. Evidently, the value of turbulent diffusivity for sea ice is expected to differ from those used to parameterize oceanic or atmospheric eddies. The aim of this work is to find the mass balance equation accounting for deformational diffusion and to estimate the importance of deformational and turbulent diffusion to the sea ice mass balance. In the following discussion, we interchangeably use the words "floes" and "particles."

\section{Modeling and evaluating diffusional mass and momentum transfer for sea ice}

\section{a. Deformational diffusion}

Deformational mass diffusion can be formally taken into account by the introduction of the mass-weighted velocity of particles. This can be done by considering sea ice as a multicomponent fluid with a continuous diversity (Faria 2001), determined by the continuous distribution of floe thickness. If for simplicity we assume that floes possess only a discrete number of thicknesses identified by the index $i=0, \ldots, N$, then we can write the mass balance for the $i$ th component as

$$
\frac{\partial A_{i} h_{i}}{\partial t}+\operatorname{div}\left(\mathbf{v}_{i} A_{i} h_{i}\right)=A_{i} f_{i}+b_{i}
$$

where $A_{i}$ is the ice concentration, $h_{i}$ is ice thickness, $\mathbf{v}_{i}$ is the areally averaged ice velocity of the $i$ th component, $t$ is time, $f_{i}$ models thermodynamic growth, and $b_{i}$ is the mass exchange between different components during ridging $\left(\Sigma_{i} b_{i}=0\right)$. Summation over the components yields the standard-form mass balance equation

$$
\frac{\partial A h}{\partial t}+\operatorname{div}(\mathbf{u} A h)=A f,
$$

where $A \equiv \Sigma_{i} A_{i}$ is the cumulative ice concentration, $h$ 


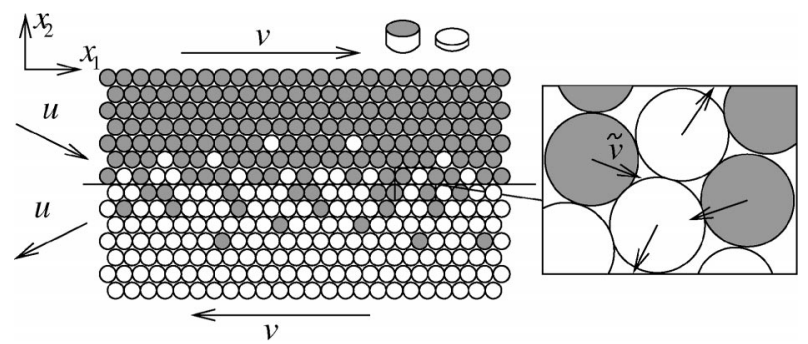

Fig. 2. Particle mixing in simple shear with a close-up showing random particle velocities at the discrete level. The mass-weighted velocity $\mathbf{u}$ has a component directed opposite to the thickness gradient, which is perpendicular to the mean flow direction $\mathbf{v}$.

$\equiv \Sigma_{i} A_{i} h_{i} / A$ is the mean ice thickness, $\mathbf{u} \equiv \Sigma_{i} \mathbf{v}_{i} A_{i} h_{i} /$ $(A h)$ is the mass-weighted velocity, and $f \equiv \Sigma_{i} A_{i} f_{i} / A$ is the mean thermodynamic growth per unit area of ice. Writing the mass balance in terms of the mass-weighted velocity $\mathbf{u}$ is common for modeling the dynamics of mixtures, where component characteristics can differ significantly. For sea ice, however, which consists of similar elements strongly interacting with each other, it is not practical because such a mass-weighted velocity cannot explicitly describe the real mean velocity of floes $\mathbf{v} \equiv \Sigma_{i} A_{i} \mathbf{v}_{i} / A$, which is usually measured. To illustrate this, consider simple shear of two bands of cylindrical particles on a horizontal plane. Initially, those with high mass (black) occupy $x_{2}>0$ and those with low mass (white) occupy $x_{2}<0$; see Fig. 2. Although the number of particles crossing the central line $x_{2}=0$ because diffusion in both directions is equal, there are more lighter particles moving in the positive $x_{2}$ direction than in the negative direction because of the initial distribution of particles across the central line. Therefore, the mass-weighted velocity $\mathbf{u}$ has a component in the negative $x_{2}$ direction, opposite to the particle mass gradient, and perpendicular to the continuum velocity $\mathbf{v}$. To show this mathematically, let us find the mean mass-weighted velocity in simple shear of a band of particles moving along $x_{1}$ between $x_{2}=0$ and $x_{2}=1$. We consider the situation in which there are only two types of particles present, with thicknesses $h$ and $\chi h, \chi=$ const, whose concentration is given by $c_{m}=x_{2}$ and $c_{\chi m}=1-x_{2}$, respectively. This gives the mean-thickness gradient along $x_{2}$ as $h(1-\chi)$. Because simple shear is independent of $x_{1}$, we consider only the $x_{2}$ component of a particular particle velocity $\tilde{v}_{2}=v_{2}+v_{2}^{\prime}$, where the continuum velocity $v_{2}$ plays the role of the mean particle velocity, while $v_{2}^{\prime}$ is the random deviation due to local discrete motion (e.g., Colony and Thorndike 1985). As mentioned before, this local discrete motion is caused by the random configuration of adjacent particles; see the close-up of Fig. 2. Across the whole flow band the velocity fluctuation integrates to zero

$$
\int_{0}^{1} v_{2}^{\prime} d x_{2}=0
$$

The mean mass-weighted velocity $u_{2}$ across the flow band can then be written as

$$
\begin{aligned}
u_{2} & =\left[\int_{0}^{1}\left(c_{m}+\chi c_{\chi m}\right) d x_{2}\right]^{-1} \int_{0}^{1}\left(c_{m}+\chi c_{\chi m}\right) v_{2}^{\prime} d x_{2} \\
& =\frac{2(1-\chi)}{(1+\chi)} \int_{0}^{1} x_{2} v_{2}^{\prime} d x_{2}
\end{aligned}
$$

For particles of the same thickness, $\chi=1, u_{2}=0$. If, as a very simple model for $v_{2}$, we assume that two adjacent particles exchange their positions along $x_{2}$, then, since the weighting coefficient $x_{2}$ in the above integral attributes greater weighting to the particle with larger $x_{2}$ moving in the negative $x_{2}$ direction, the whole integral is negative. Therefore, a negative thickness gradient, $\chi>1$, determines $u_{2}>0$, while a positive thickness gradient, $\chi<1$, determines $u_{2}<0$.

We ask the following: For sea ice, with what accuracy can the mass balance be written in terms of the mean velocity and thickness and not the mass-weighted velocity? In order to answer this, we write the mass balance in terms of mean fields, which introduces a mass diffusion term describing mixing of floes of different thicknesses. Then we find the accuracy with which it is possible to write the mass balance in standard form (2) in terms of the mean velocity $\mathbf{v}$.

Any boundary separating two volumes of material in a Lagrangian coordinate system is called a material boundary. In an Eulerian coordinate system, the material boundary is identified by the absence of the mass flux across it. In the case of sea ice, which is modeled as a two-dimensional continuum, the material boundary is a material line. The mass flux through a general (nonmaterial) line with the normal $\mathbf{n}$ is $\rho A h\left(\mathbf{u} \cdot \mathbf{n}-\boldsymbol{v}_{l}\right)$, where $v_{l}$ is the rate of advance of the line and $\rho$ is the ice density. The evolution of a material line is therefore described by the condition that the rate of its advance is equal to the normal component of the mass-weighted velocity, $\mathbf{u} \cdot \mathbf{n}$. Conservation of mass within an area bounded by a material line (and use of the divergence theorem) yields the mass balance (2) in the usual way. As was noted before, the mass-weighted velocity $\mathbf{u}$ differs from the mean floe velocity $\mathbf{v}$, which is obtained from observations and used in existing sea ice models. We define the areal line as the line determined by there being zero mean floe area flux across it. The evolution of the areal line, is given by its rate of advance being equal to the normal component of the mean floe velocity $\mathbf{v} \cdot \mathbf{n}$. There is zero net area of floes transferred across the areal line, but, when there is a thickness gradient across the line, floes of the same area moving in opposite directions across the line will transfer mass across the line. Because of the stochastic nature of this transfer, we model this mass transfer as diffusion. If it were possible to track individual floes, clearly this would not be necessary. To treat deformational diffusion caused by motion of particles past each other, we ignore con- 
vergence causing ridging and lead formation because this does not lead to floe mixing. The direction of the diffusion can be identified by considering a simplified situation depicted in Fig. 1: Several rows of particles perpendicular to the principal axis (the $x_{1}$ axis) of the compressive strain rate in pure shear merge to generate fewer rows. Such a process is possible only when some particles cross the areal symmetry line in both directions changing the thickness distribution along $x_{1}$, which demonstrates deformational diffusion of thickness. During this redistribution, particles move past each other (see close-up of Fig. 2). Although the morphology of leads and ridges created by sea ice deformation can determine a preferred direction of motion (e.g., Coon et al. 1998; Hibler and Schulson 2000), here we consider regions that are on average isotropic and assume that the precise configuration of adjacent particles is random so that the direction of their relative motion is also random. Therefore the diffusion is independent of the principal direction of the strain rate and is isotropic. The assumption of isotropy has also been used by Colony and Thorndike (1985) and Thorndike (1986). Note that, while diffusion is isotropic, the relative displacement of adjacent particles is strongly correlated. Generally, the correlation between relative particle displacement rapidly decreases with distance between the particles. Isotropy implies that the relative displacement parallel and perpendicular to a line connecting particles is independent of the line orientation. However, the relative displacement along and across the line connecting particles may differ because correlation between relative displacements decreases with distance.

Let us now turn to a continuum treatment of the deformational diffusive mass flux. As mentioned above, we assume that divergence is irrelevant to diffusion, because the particles do not move past each other, and consider shearing deformation only. We choose a coordinate system given by two areal lines that are directed along the principal axes of the strain rate tensor at $t=$ 0 to form an orthogonal Cartesian system $\left\{x_{1}, x_{2}\right\}$ with $x_{1}$ directed along the negative principal strain rate. After the small period of time $\Delta t$, the angle between the axes changes by $O\left(\Delta t^{2}\right)$, and so to leading order we treat the coordinate system as orthogonal. Consider a small square continuum region of sea ice whose center coincides with the origin of the coordinate system (Fig. $3)$. After a compressive deformation along $x_{1}$ and elongation along $x_{2}$, the square region becomes a rectangular one, and during this deformation some of the particles from either side of the axis $x_{2}$ move across it because of deformational diffusion. We define $c\left(x_{1}, A, h\right)$ to be the areal fraction of particles at distance $x_{1}$ from the axis $x_{2}$ that cross the $x_{2}$ axis during a unit deformation such that, if the particles cross the $x_{2}$ axis from $x_{1}<0$, then $c$ is positive, and, if from $x_{1}>0$, then $c$ is negative. For example, if all the particles located at a particular position $x_{1}=X<0$ move through the $x_{2}$ axis, then $c(X)=1$; if the fractional area of particles crossing the

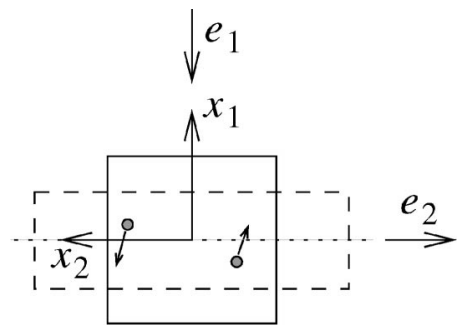

FIG. 3. Deformation of a small square continuum region around an areal line, $x_{1}=0$.

$x_{2}$ axis from position $x_{1}=\bar{X}>0$ is only $10 \%$, then $c(\bar{X})=-0.1$. The areal concentration of all particles is the fraction of local area covered by ice, $A$, which is proportional to the total number of particles in a unit region with the factor equal to the mean particle area. With these definitions, then $\left|e_{1}\right|\left[A c\left(x_{1}, A, h\right)+o(\Delta t)\right]$ is the areal concentration of particles with the coordinate $x_{1}$ that move across the axis $x_{2}$ during the principal deformation $e_{1}=\dot{e}_{1} \Delta t$. Here, $\dot{e}_{1}$ is the principal strain rate evaluated at $t=0$, and $\Delta t$ is the duration of the deformation. The term $o(\Delta t)$ appears because of the neglect of terms in the Taylor expansion of order higher than $\Delta t$. Since the axis $x_{2}$ is an areal line, the cumulative area of particles crossing it is zero,

$$
\int_{-\infty}^{+\infty} A c\left(x_{1}, A, h\right) d x_{1}=0 .
$$

The function $c\left(x_{1}, A, h\right)$ is assumed to be a strongly decreasing one with regard to $x_{1}$; that is, the fractional area of particles that move across the $x_{2}$ axis decreases strongly with increasing distance from the axis. The mass flux through a unit-length segment of the axis $x_{2}$ is determined as $q_{1}=\left|\dot{e}_{1}\right| \int_{-\infty}^{+\infty} h \rho A c d x_{1}$. The function $c$ is expected to be zero out of a small region $-x_{\max }<$ $x_{1}<x_{\max }$, where the global variables can be linearly approximated. Using linear approximations for $h$ and $A$ in terms of $x_{1}$, and using (5), we derive

$$
\begin{aligned}
q_{1}= & \rho \frac{\partial h(0)}{\partial x_{1}}\left|\dot{e}_{1}\right| A(0) \int_{-x_{\max }}^{x_{\max }} x_{1} c\left[x_{1}, A(0), h(0)\right] d x_{1} \\
& +O\left(x_{\max }^{3}\right) .
\end{aligned}
$$

Denoting

$$
\begin{aligned}
\xi & {[A(0), h(0)] } \\
& =-\int_{-x_{\max }}^{x_{\max }} x_{1} c\left[x_{1}, A(0), h(0)\right] d x_{1}>0
\end{aligned}
$$

and neglecting the higher-order terms, we derive for the mass flux along $x_{1}$

$$
q_{1}=-\rho\left|\dot{e}_{1}\right| A \xi(A, h) \frac{\partial h}{\partial x_{1}} .
$$

Because of isotropy, the mass flux along $x_{2}$ will be 


$$
q_{2}=-\rho\left|\dot{e}_{1}\right| A \xi(A, h) \frac{\partial h}{\partial x_{2}} .
$$

In an arbitrary coordinate system,

$$
\mathbf{q}=-\frac{1}{2} \rho \dot{e}_{I I} A \xi(A, h) \nabla h,
$$

where $\dot{e}_{\mathrm{II}}=2 \sqrt{1 / 2 \dot{\epsilon}_{i j}^{2}-1 / 4 \dot{e}_{I}^{2}}=\dot{e}_{1}-\dot{e}_{2}$ is the second strain-rate invariant (doubled maximum shear deformation rate) and $\dot{e}_{I}=\dot{\epsilon}_{i i}=\dot{e}_{1}+\dot{e}_{2}$ is the first strainrate invariant (divergence). While the exact formula for the principal strain rate is $\dot{e}_{1}=1 / 2\left(\dot{e}_{I}-\dot{e}_{I I}\right)$, we have discarded the first invariant in (10) because divergence is assumed to be irrelevant to the deformational diffusion.

Since the rate of advance of the areal line is $\mathbf{v} \cdot \mathbf{n}$, the diffusive mass flux through it is $(\mathbf{u}-\mathbf{v}) \cdot \mathbf{n} \rho A h$ and we can write the diffusional mass flux vector as $\mathbf{q}=$ $(\mathbf{u}-\mathbf{v}) \rho A h$. Regrouping the terms within the divergence operator of the mass balance (2) as $\mathbf{v} A h+\mathbf{q}$ and expressing the diffusive mass flux through (10), we can write the mass balance of sea ice as

$$
\begin{aligned}
\frac{\partial A h}{\partial t} & +\mathbf{v} \cdot \nabla(A h)+\dot{e}_{I} A h \\
& =\frac{1}{2} \operatorname{div}\left[\dot{e}_{I I} \xi(A, h) A \nabla h\right]+A f,
\end{aligned}
$$

where $\mathbf{v}$ is the mean velocity. The first two terms are the material time derivative, showing the change of mass per unit area at a Lagrangian point of the continuum. The third term describes the change of the ice mass per unit area due to ridging and lead formation. The first term on the right-hand side describes the total deformational diffusive mass flux, while the last term models the thickness change due to thermodynamic processes. If the material is incompressible and the thermodynamic source $f$ is zero, then the change of the thickness at a moving point will be determined only by the deformational diffusion.

In order to estimate the importance of deformational mass diffusion on the whole mass balance, we evaluate the magnitude of the diffusivity coefficient $\xi$. We can write (7) as $\xi=\overline{|c|} \bar{x}$, where $\overline{|c|}=\int_{-x \max }^{x \max } \mid c\left[x_{1}, A(0)\right.$, $h(0)] \mid d x_{1}$, and $\bar{x}=|c|{ }^{-1} \int_{-x \max }^{x \max } x c[x, A(0), h(0)] d x>$ 0 is the mean distance between a particle crossing the areal line and the line itself. The mean area fraction of particles crossing the areal line is $\overline{|c|} /\left(2 x_{\max }\right)$. The maximal distance from the line, $x_{\max }$, was chosen from the condition $c[x, A(0), h(0)]=0$ for $|x|>x_{\max }$. On the other hand, as was noted before, the function $c$ is a rapidly decreasing one, and for the purpose of finding the typical value of $\xi$, it is sufficient to consider the region $|x| \leq x_{t} \leq x_{\max }$, where $|c| \sim 1$, and the latter condition determines $x_{t} \sim \bar{x}$. Then from (7) we estimate $\xi \sim \bar{x}^{2}$, and $\xi$ represents the squared typical distance between an areal line and a particle crossing it during a unit deformation. In order to estimate the length scale of this distance, we, first, consider a unit deformation of only two rows of particles with contraction across them and extension along them. This shear deformation is representative of all deformations leading to deformational diffusion as convergence and divergence without shear will not lead to appreciable mixing. After such a deformation these two rows will merge into one 2 times as long. For a unit deformation of many rows of particles, the process is more complicated. However, most rearrangement will occur among adjacent merging rows with the distance between the centers of their particles given by the particles diameter $r$ so that $\bar{x} \sim x_{t}$ $\sim r$. Where deformational diffusion is important, the areal concentration of floes is typically high, for example, $A \approx 0.8$, and experiments with densely packed checkers, for example, show that convoluted particle paths have a low probability. Because a floe or floe aggregate size ranges from 0.1 to $10 \mathrm{~km}$, here we adopt a typical value of $1 \mathrm{~km}$ that yields $\xi \approx 10^{6} \mathrm{~m}^{2}$. Taking into account that for sea ice the typical shear strain rate is $10^{-7} \mathrm{~s}^{-1}$ (Stern et al. 1995), the deformational diffusivity coefficient $1 / 2 \dot{e}_{I I} \xi(A, h)$ can be estimated to be of order $0.05 \mathrm{~m}^{2} \mathrm{~s}^{-1}$. This value is supported by measurements of the diffusion coefficient performed on adjacent floes at a distant of $800 \mathrm{~m}$, which was found to reach $0.03 \mathrm{~m}^{2} \mathrm{~s}^{-1}$ (Legenjkov and Romanov 1972). In order to take into account the diversity of the floe and floe aggregate, sizes encountered in the Arctic, the deformational diffusion coefficient should be considered to vary within a factor of 100 of the above estimate.

As was shown by Thorndike (1986), diffusion can contribute significantly to the displacement of floes on the time scale of a floe life, for example, 10 years. However, because the change of the ice thickness is determined by several processes, the real influence of mass diffusion during this time scale is determined by its relative importance with regard to other effects. Here we examine the influence of dynamic processes, where the main mechanism changing the ice mass per unit area is divergence. As the measure of the relative contribution of divergence to diffusion, we consider their typical ratio, $\left[e_{I I}\right] \xi /\left(2\left[e_{I}\right][x]^{2}\right)$, where the brackets define typical scales. This ratio can also be interpreted as the ratio of the typical time scale of divergence to that of diffusion. The spatial scale $[x]$ appears from the second derivative of the thickness and, therefore, is a span over which the ice thickness changes considerably. For all our estimates in this work we adopt the spatial scale for the ice thickness change as the decorrelation length determined by Flato (1998). This was found by analyzing ice thickness autocorrelation between different positions using thickness estimates from upward-looking sonar measurements presented by Wadhams (1981). For thin ice of thickness $1 \mathrm{~m}$ this length scale is $200 \mathrm{~km}$, for thick ice of 5-m thickness it is $700 \mathrm{~km}$, and for typical ice thicknesses of 2-3 m we estimate it as $400 \mathrm{~km}$. The same length scale is the distance between the center of the Beaufort gyre and the sea ice margin near Alaska's 
coast. The magnitude of divergence and shear is highly variable. Interpretation of satellite data (Stern et al. 1995) show a normal distribution of divergence and shear. The divergence has a mean of $-0.04 \%$ day $^{-1}$ with standard deviation $0.38 \%$ day $^{-1}$; the shear mean and standard deviation are about $1 \%$ day $^{-1}$. From buoy data, Thomas (1999) found double the standard deviation of divergence and the mean shear between $1 \%$ day $^{-1}$ and $2 \%$ day $^{-1}$. The time scale of independent deformational fluctuations is determined by synoptic-scale atmospheric events and is estimated as 5 days (Thorndike 1986). Although during this time scale, the ratio of shear to divergence can vary from zero to infinity, the typical value of either shear or divergence is given by the larger of its mean and standard deviation. This means that on a time scale of 5 days, the typical values of divergence and shear can be taken to be about the same and the relative contribution of deformational diffusion to the areal mass change is given by $\xi /\left(2[x]^{2}\right) \approx 3 \times 10^{-6}$ for $\xi \approx 10^{6} \mathrm{~m}^{2},[x]=4 \times 10^{5} \mathrm{~m}$ with typical floe size of $1 \mathrm{~km}$. This is negligible and, even for floes of $10 \mathrm{~km}$, the relative contribution of deformational diffusion to areal mass change is $3 \times 10^{-4}$, which is negligible.

On a time scale much larger than that of independent fluctuations, the areal change of a sea ice region is determined by the mean values of strain rate as short-timescale fluctuations cancel each other out. Averaged over this time scale, much longer than 5 days, many sea ice regions move mainly due to shear. The mean value of deformations given above (Stern et al. 1995; Thomas 1999) give the ratio of the mean shear to convergence in the Arctic as about 20. The ice moves along Alaska's coast mainly because of shearing (Serreze et al. 1989), which yields a ridging intensity there that is about $1 / 20$ of that near the Canadian Arctic Archipelago (Hibler et al. 1974). This implies that on the large time scale the ratio of shear to divergence in the Beaufort Gyre $\left[e_{I I}\right] /\left[e_{I}\right] \approx 20$, and, again using $\xi \approx 10^{6} \mathrm{~m}^{2}$ for $1-\mathrm{km}$ wide floes, we find that the ratio of deformational diffusion to divergence is about $10^{-4}$. For floes of $10-\mathrm{km}$ size, deformational diffusion is 100 times as large and reaches $1 \%$ of divergence, but its neglect is still an acceptable error. We thus conclude that on all time scales there is negligible influence of deformational diffusion on the mass balance. Therefore the diffusion term in (11) can be safely neglected, after which the mass balance equation (11) written in terms of mean velocity attains the same form as that written in terms of massweighted velocity (2).

\section{b. Turbulent diffusion}

Turbulent eddies in the atmosphere and ocean exert drags on the ice cover that cause mixing of different sea ice regions. We model this mixing as turbulent diffusion. For short-time-scale prediction, of say 5 days, the boundary and initial conditions for modeling atmospheric processes are derived from observations and atmospheric events with a scale larger than the grid resolution can be predicted with reasonable accuracy. For much longer time scale prediction, atmospheric flow instability and lack of observations of the evolution of the boundary conditions mean that large-scale atmospheric eddies cannot be correctly described by models, even if they are run on small time and grid steps. Such events should be modeled in terms of turbulent diffusion of mass and momentum, and as the spatial scale for long-time-scale processes we adopt $400 \mathrm{~km}$ as before. In order to estimate the importance of turbulent diffusion, we introduce for simplicity the partial ice thickness $h_{p}=A h$ (Gray and Morland 1994), which is the thickness of a continuous layer that has the same ice volume as the ice pack. Neglecting deformational diffusion, we write the mass balance as

$$
\frac{\partial h_{p}}{\partial t}+\operatorname{div}\left(\mathbf{v} h_{p}\right)=f_{p} .
$$

After performing Reynolds-averaging or filtering, this becomes

$$
\frac{\partial H_{p}}{\partial t}+\operatorname{div}\left(\mathbf{V} H_{p}\right)=\operatorname{div} \mathbf{Q}+F_{p},
$$

where the capital letters denote the ensemble-averaged (Reynolds averaged) or filtered variables [as used in large-eddy simulation (LES)], the bar denotes averaging or filtering, and $\mathbf{Q}=\mathbf{V} H_{p}-\overline{\mathbf{v} h_{p}}$ is the turbulent or subgrid ice thickness flux (Cabot and Moin 1993), respectively. For Reynolds averaging, expanding the velocity and partial thickness about their means, $\mathbf{v}=\mathbf{V}$ $+\mathbf{v}^{\prime}, h_{p}=H_{p}+h_{p}^{\prime}$, and, since $\overline{\mathbf{V} h_{p}^{\prime}}=\overline{H_{p} \mathbf{v}^{\prime}}=\mathbf{0}$, the turbulent ice thickness flux can also be written $\mathbf{Q}=$ $-\overline{\mathbf{v}^{\prime} h_{p}^{\prime}}$ (for spatial filtering in LES, this does not hold). In order to estimate the influence of the turbulent diffusion term $\mathbf{Q}$, we use the standard gradient-type relation $\mathbf{Q}=-\zeta \nabla H_{p}$, where $\zeta$ is the coefficient of turbulent diffusivity with size dependent upon the unresolved scales. Finding the form and the magnitude of the turbulent diffusivity for sea ice theoretically is a difficult problem beyond the scope of this article. Therefore, we use empirical values found in field measurements in the Arctic basin in 1966 for time scales from 0.5 to 5 days and spatial scales from 10 to $800 \mathrm{~km}$ (Gudkovich 1979). The turbulent diffusivity coefficient for the largest spatial scale was obtained by measuring displacements between the stations, North Pole-15 and North Pole-13. It was found that, for scales of more than $100 \mathrm{~km}$, the diffusivity follows the Richardson-Obukhov dependence on typical length scale $l$ as $\zeta \propto l^{4 / 3}$ with the coefficient of proportionality dependent on the turbulent kinetic energy dissipation. Martin and Thorndike (1985) found a similar type of relation with the exponent magnitude lying in the range from 1.1 to 1.3 . From Gudkovich's measurements, for scales of order $100 \mathrm{~km}, \zeta$ $\approx 14 \mathrm{~m}^{2} \mathrm{~s}^{-1}$, while for length scales $300-800 \mathrm{~km}$, the turbulent diffusivity coefficient was found to be 70-256 
$\mathrm{m}^{2} \mathrm{~s}^{-1}$. From Thorndike (1986), the mean squared relative displacement parallel to a $400-\mathrm{km}$ separation was measured to be $12 \mathrm{~km} \mathrm{day}^{-1}$, which corresponds to the lower estimate of a diffusion coefficient of $70 \mathrm{~m}^{2} \mathrm{~s}^{-1}$ using random walk theory. As a compromise between the data reported in Gudkovich (1979) and Thorndike (1986), for a 400-km length scale we adopt $\zeta \approx 100$ $\mathrm{m}^{2} \mathrm{~s}^{-1}$.

We are interested here in comparing the effects of divergence and turbulent diffusion on the partial ice thickness change. Although at a particular time their ratio is highly variable, for our estimates we use their typical values as generally representing sea ice dynamical processes, $\zeta /\left(\left[\dot{e}_{I}\right][x]^{2}\right)$. As mentioned earlier, in estimating the strain rate magnitude we distinguish between two time scales: 5 days (day-to-day modeling), the typical duration of independent synoptic events with $\left[\dot{e}_{I}\right] \approx 1 \%$ day $^{-1} \approx 10^{-7} \mathrm{~s}^{-1}$, and a time scale much larger than 5 days where the independent events balance each other out to determine the deformation given by its mean value, $\left[\dot{e}_{I}\right] \approx 0.04 \%$ day $^{-1} \approx 5 \times 10^{-9} \mathrm{~s}^{-1}$ (Stern et al. 1995). Regardless of the magnitude of unresolved scales considered, the length scale $[x]$ is determined by the span of significant ice thickness change and is taken as $400 \mathrm{~km}$. For day-to-day modeling ([t] $\approx 5$ days) with unresolved scale of turbulent motion of about $100 \mathrm{~km}\left(\left[\dot{e}_{I}\right] \approx 10^{-7} \mathrm{~s}^{-1}, \zeta \approx 14 \mathrm{~m}^{2} \mathrm{~s}^{-1}\right)$, the ratio of turbulent diffusion to divergence $\zeta /\left(\left[\dot{e}_{I}\right][x]^{2}\right)$ is estimated as $10^{-3}$ and is thus negligible. For large-timescale modeling ( $[t] \gg 5$ days), with spatial scale for turbulent diffusion of $400 \mathrm{~km}\left(\left[\dot{e}_{I}\right] \approx 5 \times 10^{-9} \mathrm{~s}^{-1}, \zeta\right.$ $\approx 100 \mathrm{~m}^{2} \mathrm{~s}^{-1}$ ), the ratio is estimated as 0.12 . If an even larger spatial scale of sea ice turbulent motion is taken as $800 \mathrm{~km}$, then from Gudkovich (1979) and Thorndike (1986) we infer $\zeta \approx 400 \mathrm{~m}^{2} \mathrm{~s}^{-1}$, and $\zeta /\left(\left[\dot{e}_{I}\right][x]^{2}\right)$ is estimated as 0.25 . Therefore the effect of turbulent diffusion on the sea mass balance can exceed $10 \%$ of the effect of divergence for large time-scale modeling where the turbulent motion is not resolved on the basin scale.

If we write the momentum balance for sea ice through a set of equations for every component identified by mean floe thickness, then, after summation of these equations, we arrive at the momentum balance in terms of the mean mass-weighted velocity. This momentum balance includes, in addition to the sum of the stresses acting on every component, an additional stress (diffusional momentum transfer) due to the nonlinearity of the inertial terms. In order to write the momentum balance through the mean velocity, these terms should strictly be treated. However, because the inertial stress is negligible for sea ice, being typically of order 0.01 $\mathrm{N} \mathrm{m}^{-1}$ as compared with internal ice stress caused by wind forcing of order $10^{5} \mathrm{~N} \mathrm{~m}^{-1}$ (e.g., Steele et al. 1997), this inertial effect may be safely neglected.

\section{Conclusions}

We have presented a discussion that demonstrates that deformational and turbulent mass and momentum dif- fusion in sea ice, caused by deformation-induced mixing and unresolved scales of turbulent atmospheric and oceanic motion, affects the general form of the mass and momentum balance equations. The deformational mass transfer is estimated to be negligible for sea ice, which also means that the mass balance can be written in terms of the mean velocity rather than mean mass-weighted velocity. However, for the Beaufort gyre the effect of turbulent mass diffusion on the dynamic partial ice thickness redistribution can exceed $10 \%$ of the divergence effect if basin-scale atmospheric and oceanic eddies are not resolved in large time-scale modeling. When the unresolved scales are of order $100 \mathrm{~km}$ and 5 days, the error is estimated as less than $0.1 \%$. The diffusional momentum transfer can be disregarded, because inertial effects are much smaller than the other stresses determining sea ice motion.

Acknowledgments. We thank the anonymous referees for useful comments on an earlier draft. This work is funded by NERC.

\section{REFERENCES}

Cabot, W., and P. Moin, 1993: Large eddy simulation of scalar transport with the dynamic subgrid-scale model. Large Eddy Simulation of Complex Engineering and Geophysical Flows, B. Galperin and S. A. Orszag, Eds., Cambridge University Press, 141158.

Colony, R., and A. S. Thorndike, 1985: Sea ice motion as a drunkard's walk. J. Geophys. Res., 90, 965-974.

Coon, M. D., G. A. Maykut, R. S. Pritchard, D. A. Rothrock, and A. S. Thorndike, 1974: Modelling the pack ice as an elastic-plastic material. AIDJEX Bull., 24, 1-105.

— - G. S. Knoke, D. C. Echert, and R. S. Pritchard, 1998: The architecture of an anisotropic elastic-plastic sea ice mechanics constitutive law. J. Geophys. Res., 103, 21 915-21925.

Faria, S. H., 2001: Mixtures with continuous diversity: General theory and application to polymer solution. Contin. Mech. Thermodyn., 13, 91-120.

Flato, G. M., 1998: The thickness variable in sea-ice models. Atmos.Ocean, 36, 29-36.

_ - and W. D. Hibler III, 1992: On modeling pack ice as a cavitating fluid. J. Phys. Oceanogr., 22, 626-651.

Gray, J. M. N. T., and L. W. Morland, 1994: A two-dimensional model for dynamics of sea ice. Philos. Trans. Roy. Soc. London, A347, 219-290.

Gudkovich, Z. M., 1979: K izucheniju turbulentnogo dvizhenija ledjanogo pokrova $\mathrm{v}$ arkticheskom bassejne (On study of turbulent motion of ice cover in Arctic basin). Tr. Arkt. i Antark. Inst., 364, 34-39.

Hibler, W. D., III, 1979: A dynamic thermodynamic sea ice model. J. Phys. Oceanogr., 9, 815-846.

, and E. M. Schulson, 2000: Anisotropic failure and flow of flawed sea ice. J. Geophys. Res., 105, 17 105-17 120.

_ S. J. Mock, and W. B. Tucker, 1974: Classification and variation of sea ice ridging in the western Arctic basin. J. Geophys. Res., 79, 2735-2743.

Holland, D. M., 2001: An impact of subgrid scale ice-ocean dynamics on sea-ice cover. Climate Dyn., 14, 1585-1601.

Legenjkov, A. P., and M. A. Romanov, 1972: O podvizhkah ljda v Arkticheskom bassejne (On ice motion in Arctic basin). Okeanologija, 12, 617-624.

Litinsky, V. A., 1968: Obnaruzhenije ostatkov amerikanskoj drejfujushej stanzii "Charley" v rajone k severu ot o. Vrangelja (Dis- 
covery of remains of American drifting station "Charley" north of Wrangel Island). Probl. Arkt. i Antarkt., 28, 140-145.

Martin, S., and A. S. Thorndike, 1985: Dispersion of sea ice in Bering Sea. J. Geophys. Res., 90, 7223-7226.

Serreze M. C., R. G. Barry, and A. S. McLaren, 1989: Seasonal variations in sea ice motion and effects on sea ice concentration in the Canada Basin. J. Geophys. Res., 94, 10 955-10 970.

Steele, M., J. Zhang, D. A. Rothrock, and H. Stern, 1997: The force balance of sea ice in a numerical model of the Arctic Ocean. $J$. Geophys. Res., 102, 21 061-21 079.

Stern, H. L., D. A. Rothrock, and R. Kwok, 1995: Open water pro- duction in Arctic sea ice: Satellite measurements and model parameterizations. J. Geophys. Res., 100, 20 601-20 612.

Thomas, D., 1999: The quality of sea ice velocity estimates. J. Geophys. Res., 104, 13 627-13 652

Thorndike, A. S., 1986: Diffusion of sea ice. J. Geophys. Res., 91, 7691-7696.

Tremblay, L. B., and L. A. Mysak, 1997: Modeling sea ice as a granular material including the dilatancy effect. J. Phys. Oceanogr., 27, 2342-2360.

Wadhams, P., 1981: Sea-ice topography of the Arctic Ocean in the region $70^{\circ} \mathrm{W}$ to $25^{\circ} \mathrm{E}$. Philos. Trans. Roy. Soc. London, A302, $45-85$. 